Instituto Internacional de Investigación y Desarrollo Tecnológico Educativo INDTEC, C.A.

DOI: https://doi.org/10.29394/Scientific.issn.2542-2987.2021.6.20.7.130-151

OAI-PMH: http://www.indteca.com/ojs/index.php/Revista Scientific/oai

Artículo Original / Original Article

\title{
Teletrabajo en tiempos de pandemia: Un reto laboral en la educación superior
}

Autores: Joanna Carolina Ramírez Velásquez Universidad Católica de Cuenca, UCACUE joanna.ramirez@ucacue.edu.ec Cuenca, Ecuador https://orcid.org/0000-0003-3266-7195

Luis Ernesto Quinde Quizhpi Universidad Católica de Cuenca, UCACUE lequindeq@ucacue.edu.ec Cuenca, Ecuador https://orcid.org/0000-0001-8348-3068

Ricardo Agustín Alarcón Vélez Universidad Católica de Cuenca, UCACUE ricardo.alarcon@ucacue.edu.ec Cuenca, Ecuador https://orcid.org/0000-0002-1910-8527

Cesar Remigio Vega Abad Universidad Católica de Cuenca, UCACUE crvegaa@ucacue.edu.ec

Cuenca, Ecuador https://orcid.org/0000-0003-3301-2668

\section{Resumen}

El teletrabajo es una forma de organización laboral que se desarrolla bajo el uso esencial de la tecnología, ante la pandemia del COVID-19 se ha constituido como una herramienta clave para la educación superior. El objetivo de esta investigación fue analizar las repercusiones laborales, sociales y tecnológicas del COVID-19 en el teletrabajo y las directrices para su implementación frente la pandemia. La metodología utilizada consistió en una investigación de tipo exploratoria, por cuanto, existe poca literatura reportada sobre el tema de estudio, descriptiva y para la recolección y análisis de la información se recurrió a la revisión de las bases de datos: SciELO, Latindex, Scopus, Redalyc, Dialnet, durante los últimos cinco años, además de otras fuentes documentales nacionales e internacionales. La educación superior pública y privada implementó el teletrabajo para garantizar el derecho constitucional a la educación y dar continuidad a las relaciones de trabajo, enfrentando barreras de tipo laboral, social, tecnológico y familiar generadas por la pandemia. La implementación del teletrabajo para la educación a distancia en pandemia requiere el establecimiento de directrices que permitan obtener resultados favorables para Instituciones, docentes y estudiantes, por tanto, deben realizarse estudios que involucren a todos los actores sociales.

Palabras clave: trabajo; pandemia; enseñanza superior; tecnología educacional. Código de clasificación internacional: 5802.04 - Niveles y temas de educación.

\section{Cómo citar este artículo:}

Ramírez, J., Quinde, L., Alarcón, R., \& Vega, C. (2021). Teletrabajo en tiempos de pandemia: Un reto laboral en la educación superior. Revista Scientific, 6(20), 130-151, e-ISSN: 2542-2987. Recuperado de: https://doi.org/10.29394/Scientific.issn.2542-2987.2021.6.20.7.130-151

Fecha de Recepción: 15-01-2021
Fecha de Aceptación: 13-03-2021
Fecha de Publicación: 05-05-2021 


\title{
Teleworking in times of pandemic: A job challenge in higher education
}

\begin{abstract}
Teleworking is a form of labor organization that is developed under the essential use of technology, in the face of the COVID-19 pandemic it has become a key tool for higher education. The objective of this research was to analyze the labor, social and technological repercussions of COVID-19 on teleworking and the guidelines for its implementation in the face of the pandemic. The methodology used consisted of an exploratory research, since there is little literature reported on the subject of study, descriptive and for the collection and analysis of the information, a review of the databases was used: SciELO, Latindex, Scopus, Redalyc, Dialnet, during the last five years, in addition to other national and international documentary sources. Public and private higher education implemented teleworking to guarantee the constitutional right to education and to give continuity to work relationships, facing labor, social, technological and family barriers generated by the pandemic. The implementation of teleworking for distance education in a pandemic requires the establishment of guidelines that allow obtaining favorable results for Institutions, teachers and students, therefore, studies that involve all social actors must be carried out.
\end{abstract}

Keywords: job; pandemic; higher level education; educational technology.

International classification code: 5802.04 - Levels and subjects of education.

\footnotetext{
How to cite this article:

Ramírez, J., Quinde, L., Alarcón, R., \& Vega, C. (2021). Teleworking in times of pandemic: A job challenge in higher education. Revista Scientific, 6(20), 130-151, e-ISSN: 2542-2987. Recovered from: https://doi.org/10.29394/Scientific.issn.2542-2987.2021.6.20.7.130-151
}

Date Received: 15-01-2021
Date Acceptance: 13-03-2021
Date Publication: 05-05-2021 


\section{Introducción}

Ante la pandemia ocasionada por la enfermedad del coronavirus (2019$\mathrm{nCoV}$ ) anunciada por la Organización Mundial de la Salud como una emergencia internacional de salud pública, el Ecuador a través del Decreto emitido por Moreno (2020): como estado de excepción por calamidad pública, estableciendo dentro de sus acciones la suspensión de la jornada presencial laboral y promoviendo la implementación del teletrabajo para el sector público y privado.

El teletrabajo se ha caracterizado como toda actividad laboral bajo relación de dependencia a través del uso de las herramientas tecnológicas, siempre que las actividades se realicen en un lugar distinto al centro de trabajo u oficinas del empleador.

En Latinoamérica, el teletrabajo iniciaba su implementación, según la Academia Internacional de Teletrabajo (2017): en Chile se inició entre los años 2017-2018 en el Instituto Nacional de Propiedad Intelectual con el $10 \%$ de los trabajadores, en Brasil estiman que el 16, $2 \%$ de trabajadores lo aplican. En Colombia, para Tapasco-Alzate y Giraldo-García (2020): en el año 2018, la cifra de teletrabajadores fue de 122.278. En el Ecuador de acuerdo con indicadores laborales del Ministerio del Trabajo (MDT, 2020a): el teletrabajo emergente hasta octubre del año 2020 alcanzó para el sector público 286.401 trabajadores y en el sector privado 155.208.

De modo pues, el teletrabajo se constituye como la herramienta más idónea ante la pandemia que afecta a todos los sectores de la sociedad, y el sistema educativo es uno de los más impactados, en el cual se debe implementar este método laboral de manera inmediata, sin que pueda realizarse una planificación laboral, tecnológica, y social, debido a las medidas de aislamiento. Para Risso, et al. (2020): la pandemia precipitó la aplicación del teletrabajo, aun cuando, su implementación no es nueva en el mercado laboral, la emergencia sanitaria determinó que las empresas pasaran a 
trabajar desde el domicilio siempre que las actividades lo permitieran, se impuso una modalidad de trabajo que era poco desarrollada, y sobre la que muchos actores de la sociedad presentaban dudas sobre su viabilidad.

El teletrabajo plantea algunos desafíos, entre ellos, la adaptación de los horarios y del lugar de trabajo, medidas de salud y seguridad laboral que requieren una regulación jurídica bien estructurada, ya que, laborando en casa no se presentan las condiciones laborales regulares en cuanto a medidas ergonómicas, comunicación y se realizan las actividades sin control en la jornada de trabajo.

Para la implementación del teletrabajo frente al COVID-19, es necesario que el empleador público o privado establezcan las directrices internas que orienten al docente a una práctica adecuada del mismo, en el ámbito laboral, social y tecnológico dentro del marco jurídico vigente, con la finalidad de generar comportamientos de trabajo seguros y resultados positivos en su ejecución. Por ello, la importancia de realizar un estudio, ya que el teletrabajo en tiempos de pandemia se presenta bajo un contexto que genera muchos más desafíos y en el sector educativo probablemente este medio alternativo de trabajo deba permanecer por mucho más tiempo, en el caso de la educación superior actualmente se presenta como una propuesta para la educación en línea.

Así pues, este estudio es parte del proyecto de investigación denominado Directrices para el teletrabajo del personal docente de la Universidad Católica de Cuenca frente al COVID-19, el cual fue presentado por la Unidad Académica de Ciencias Sociales, carrera de Derecho Extensión La Troncal, con el fin de formar nuevos conocimientos, permitiendo un aporte desde la formulación de las siguientes preguntas: ¿Cuáles son las repercusiones del COVID-19 en el teletrabajo?; ¿Qué directrices se deben establecer para su implementación en pandemia?.

Para responder a la interrogante, en esta investigación se planteó como 
objetivo, analizar las repercusiones laborales, sociales y tecnológicas del COVID-19 en el teletrabajo y las directrices requeridas para su implementación.

\section{Metodología}

El presente estudio se inició como una investigación de tipo exploratoria, por cuanto, al revisar la literatura reportada sobre el teletrabajo en la educación superior bajo el contexto de la pandemia, se observó que ha sido poco estudiado. Además, el alcance de la investigación fue descriptivo, se realizó una investigación teórica, documental, que consistió en la revisión bibliográfica de la literatura de artículos científicos indexados en revistas nacionales e internacionales, además de guías y protocolos emitidos para la implementación del teletrabajo en tiempos de pandemia.

En cuanto a la técnica utilizada para la recolección y análisis del tema de estudio, se planteó como propósito la revisión de la literatura reportada a través de los artículos indexados en las principales bases de datos: SciELO, Latindex, Redalyc, Dialnet, Scopus, Google Académico, dando prioridad aquellos publicados durante los últimos cinco (5) años, siendo considerados los que se relacionaban directamente con el tema de estudio, además, se analizaron otros documentos emanados de organismos internacionales, normas nacionales, guías y protocolos que tratan sobre el teletrabajo en tiempos de pandemia.

\section{Resultados}

El teletrabajo, tal como lo exponen Velásquez y Vera (2018): requiere de tres elementos esenciales, tales como:

Es un acuerdo entre empleador y colaborador para trabajar fuera de una oficina o instalación establecida. Las TIC son indispensables para la ejecución de las funciones del colaborador bajo una modalidad de teletrabajo. El teletrabajo 
puede ser ejecutado en cualquier momento, en cualquier lugar, según la disponibilidad del trabajador (pág. 42).

Este modo de prestación de servicios se caracteriza por el uso esencial de las herramientas tecnológicas en el desarrollo de sus actividades, las cuales se llevan a cabo fuera de la organización, con horarios flexibles, bajo el control y evaluación por resultados.

En la implementación del teletrabajo se requiere además las políticas de la organización laboral tendientes a lograr la aceptación de este modo de trabajo por parte de los trabajadores y empleados. Por su parte, Chávez, Martínez y Dávila (2020a): sostienen que "[...] la contingencia sanitaria ha puesto de relieve estas formas de trabajo y de enseñanza-aprendizaje [...]" (pág. 275).

Al respecto, Sánchez, Montenegro y Medina (2019): exponen que "en el país ecuatoriano, la era del conocimiento ha permitido que las empresas opten por la modalidad de teletrabajo, idea que inicia con la globalización de la economía, los cambios de la evolución tecnológica, la competitividad y la política" (pág. 94). Ante la pandemia el teletrabajo emergente se define como lo expresa el Acuerdo Ministerial del Ministerio del Trabajo (MDT, 2020b): como la forma de prestar servicios no presenciales, en jornadas de trabajo ordinarias y especiales donde el trabajador del sector público o privado se desempeña fuera de las instalaciones de la empresa.

\subsection{Repercusiones del COVID-19 en el Teletrabajo}

\subsubsection{En el ámbito laboral}

El teletrabajo es un modo de organización laboral que surgió en países desarrollados debido a la evolución de la tecnología de la información y comunicación, en Latinoamérica comenzaba su aplicación con menos impulso, sin embargo, ante las medidas de confinamiento obligatorio 
generadas por el COVID-19, esta forma de trabajo constituyó la herramienta necesaria para muchos sectores económicos y de manera muy especial para el sistema de educación superior.

Al respecto, Cóndor-Herrera (2020): refiere que la realidad generada por la crisis sanitaria ha obligado de forma abrupta a la transformación de un nuevo modelo educativo, representando un desafío para estudiantes, docentes, y autoridades, este desafío es aún mayor en Latinoamérica, por cuanto, se encuentra en un plano de desigualdad social y en cada estado deberán establecer las medidas necesarias para garantizar la educación y el trabajo, considerando que el retorno a las actividades laborales presenciales no se dará en un corto plazo.

El teletrabajo como herramienta es utilizado en muchos ámbitos públicos y privados, en la economía formal e informal, sin embargo, el teletrabajo normado en algunas legislaciones con ocasión a la pandemia, considera aquellas actividades laborales que se desarrollan bajo relación de dependencia.

Dentro de los beneficios que ofrece el teletrabajo, para Peralta, Bilous, Flores y Bombón (2020): a nivel empresarial "se tienen por visionarias, ya que brindan la opción a sus trabajadores de desempeñarse desde su casa u otro lugar de trabajo, lo que rompe los esquemas tradicionales y genera en el teletrabajador mayor confianza en la organización” (pág. 334). Sin embargo, Estévez (2020): refiere que en el teletrabajo organizado por efectos de la emergencia se encontraron perjudicados las personas adultas que reflejaban negativa en la utilización de la tecnología, aquellos que no disponen de equipos tecnológicos, un lugar apropiado y conectividad, mujeres y hombres con responsabilidades familiares, los trabajadores con actividades presenciales, además el núcleo familiar, porque el horario de trabajo de alguno de sus miembros, lleva a la desestructuración del grupo.

La sociedad se encuentra en un tiempo de innovación y transformación 
del sistema educativo, se debe pensar y analizar, no sólo en el mundo que se mueve a través de las redes, sino también, se deben considerar aquellos aspectos que actualmente demandan el mercado de trabajo, donde el docente tiene como objetivo fundamental preparar al alumnado para la vida digital. Todos estos cambios y transformaciones en la forma de impartir la educación y por ende de desarrollar las actividades académicas han constituido para el docente un verdadero reto, que van desde el efectivo acceso y disponibilidad de los medios tecnológicos hasta las destrezas para su utilización y adaptación a esta nueva forma de prestar sus servicios.

De acuerdo con ello para Chávez, Martínez y Dávila (2020b): ante las repercusiones del COVID-19 las Instituciones de Educación Superior ejecutan sus acciones en cooperación con las autoridades sanitarias, con el fin de continuar con las actividades académicas e investigación haciendo uso de las herramientas como son las aulas virtuales, repositorios, y bibliotecas digitales. Bajo el contexto de la pandemia, el teletrabajo genera aspectos positivos en el sector educativo, no se detuvo el proceso de enseñanza-aprendizaje, se mantienen las relaciones laborales, los ingresos económicos de las Instituciones, no se requiere la presencia de los docentes y trabajadores dentro de las instalaciones, generando reducción de costos en salarios, servicios y mantenimiento de infraestructuras.

No obstante, las interrogantes surgen alrededor de los teletrabajadores que se encuentran desempeñando sus actividades bajo restricciones laborales, sociales y familiares para evitar el contagio de la enfermedad, donde las jornadas de trabajo se han prolongado, no disponen de condiciones adecuadas dentro del hogar para garantizar la salud y seguridad, se incrementan los gastos por servicios públicos como electricidad, agua, acceso al internet, y se presentan mayores dificultades al no encontrar el límite entre las relaciones familiares-laborales. 


\subsubsection{En lo social}

La amplia digitalización de las diferentes actividades laborales y el acceso a internet, han provocado el distanciamiento social y por otra parte el mantenimiento de la actividad productiva, lo cual se aplicó también en el sector educativo. Para solventar en algo este problema, Quintero (2020): sostiene la necesidad de buscar estrategias de enseñanza creando lugares para las tareas, horarios y expectativas, fomentar la división de tareas complejas, ampliar los medios de comunicación docente estudiante, y una mejor preparación de la clase por el docente.

Ante lo expuesto, Montes-Rodríguez, Chen-Quezada, HernándezSánchez y Villalobos-Benavides (2020): indican que la solución al problema de fondo implica un cambio mayor a una simple facilitación de tareas, a una mejor didáctica o a la tolerancia y oportunidades para con el estudiante, pues es necesario avanzar hacia un currículum flexibles que integre nuevas propuestas formativas. Ante ello, Castellano, Almagro y Fajardo (2021): sostienen que es necesario dentro de los conocimientos empleados por el docente en el desarrollo de sus actividades establecer habilidades sociales para poder resolver las situaciones que se presenten, además de motivar al estudiante.

Existen en este mismo aspecto factores positivos como lo son la eliminación del tiempo de movilización, lo cual origina la posibilidad de compartir mayores momentos con la familia, es benéfico para la sociedad, al provocar un efecto igualador para los géneros, así antes la parte femenina debía cargar con toda la responsabilidad del hogar y los hijos, por lo que terminaba en muchos casos abandonado sus justas aspiraciones profesionales, y a la vez recayendo en la parte masculina todo el peso y preocupación del sustento del hogar.

El aislamiento social a causa del COVID-19, es uno de los aspectos que mayores repercusiones han generado en la población que se desempeña en 
teletrabajo y más aún en el sector educativo, con consecuencias para el docente no sólo en el ámbito laboral y personal, quien a fin de obtener resultados satisfactorios en el cumplimiento de sus funciones debe tener un contacto permanente con el estudiante a través de los medios tecnológicos, involucrase en aspectos familiares y sociales de éste, también debe abordar los temas que conllevan el aislamiento como la falta de contacto físico con su supervisor inmediato y compañeros de trabajo, mayor control y responsabilidad, así como, solución de problemas de tipo tecnológico, laboral, familiar, y de infraestructura.

\subsubsection{En lo tecnológico}

Una de las características fundamentales del teletrabajo es que su desarrollo requiere del uso exclusivo de los medios tecnológicos y por ello sus implicaciones en el necesario acceso a plataformas digitales y las capacidades necesarias del teletrabajador en el uso de estas.

Al respecto, Guaña-Moya, Quinatoa-Arequipa y Pérez-Fabara (2017): refieren que la formación al personal en temas informáticos y computacionales es prioritaria al momento de implementar el teletrabajo, es decir, se debe iniciar con la formación ya que, al adquirir estas capacidades tecnológicas, crecen las oportunidades hacia un nuevo modo de vida social donde prevalece la conectividad. Por otra parte, el avance del teletrabajo incrementa la necesidad de tecnologías acordes a las necesidades de las instituciones, así como, la inseguridad de la información de esta, por lo que, Conejo-Navarro (2020): expone que tanto en el sector público como en el privado las empresas que se desenvuelven a través del uso de las herramientas tecnológicas deben reforzar sus protocolos para la protección y seguridad de la información. Al respecto Chávez, Martínez y Dávila (2020c): al referirse a la educación superior y el teletrabajo en pandemia exponen que:

Para los maestros de educación superior es de gran 
importancia dar los tópicos profesionales que aportan herramientas para el desempeño profesional, a través de la modalidad a distancia se puede optar por estudios de caso, simuladores, videoconferencias, chat en vivo, foros asincrónicos, videos didácticos, entre otros (pág. 269).

En efecto, el paso de la modalidad presencial a la virtual provocada de forma inesperada como efecto de la pandemia obliga tanto a estudiantes como docentes a la adquisición de nuevas competencias tecnológicas. En relación, Acuña y Sánchez (2020), manifiestan que:

Los sistemas educativos del presente (antes y durante la pandemia) evidencian desigualdades y asimetrías sociales propias de las sociedades en las que funcionan, en el caso latinoamericano es histórica la carencia de condiciones materiales de la educación como infraestructura, equipamiento y tecnologías [...] (pág. 1283).

De modo pues, ante la forma abrupta en que se presentó la pandemia generada por el COVID-19, a nivel global y de manera más pronunciada en América Latina el acceso a la tecnología no estaba asegurado para todos los sectores y en general para toda la sociedad, el sistema educativo público y privado debió establecer estrategias para asegurar el derecho constitucional a la educación y continuar con el desarrollo de las clases de manera virtual haciendo uso de todas las herramientas tecnológicas disponibles, sin embargo, esta exigencia de acceso a la tecnología para el teletrabajo y desarrollo de las clases en línea afecta tanto a docentes como estudiantes que se encuentren con estas barreras.

\subsection{Directrices para la implementación del teletrabajo frente al COVID-19}

En el Ecuador el teletrabajo fue una de las estrategias empleadas para contrarrestar el contagio de la enfermedad causada por el coronavirus, cumplir con las medidas de aislamiento social y al mismo tiempo continuar con la vida 
laboral de docentes y empleados administrativos públicos y privados, garantizando el derecho constitucional a la educación, lo cual requiere necesariamente de directrices o instrucciones claras establecidas desde la regulación jurídica vigente.

Para hacer frente a la emergencia sanitaria en Ecuador el Ministerio del Trabajo (MDT, 2020c): dispuso la implementación del teletrabajo emergente tanto para el sector público como para el privado, expresando dicha resolución que no se deben modificar las condiciones contractuales, ni vulnerar los derechos laborales, así como, no deben originarse causas para la terminación de la relación laboral, de igual manera dispone para autoridades del sector público o empleadores del sector privado establecer directrices, así como, controlar y monitorear las actividades ejecutadas por el teletrabajador.

Así pues, además de las normas generales y especificas reguladas en cada estado, a nivel global fueron emitidas guías y protocolos para promover las buenas prácticas de esta modalidad de prestación de servicios frente al contexto del COVID-19.

En correspondencia con la Organización Internacional del Trabajo (OIT, 2020): se expidió una guía práctica con fines de establecer las recomendaciones para que el teletrabajo frente a la pandemia sea eficaz, promoviendo para ello, la motivación por parte del empleador para que el trabajador se exprese cuando exista sobre carga laboral, así como, reconocer la necesidad de desconexión del trabajador para realizar de la manera más adecuada el trabajo individual, además otorgar el reconocimiento de forma positiva al trabajo bien elaborado por el trabajador y analizar las necesidades tecnológicas, como equipos, herramientas y capacitación.

Por su parte, el Centro de Estudio del Trabajo y Factores Humanos (CETyFH-UV, 2020): emitió la guía para el teletrabajo en condiciones de pandemia COVID-19, a fin de establecer algunas recomendaciones, por cuanto, el teletrabajo bajo el contexto de la pandemia es totalmente diferente 
al teletrabajo en condiciones habituales, es por ello que, deben estar previstos los horarios, tratando de mantener un horario de oficina evitando horarios nocturnos y fines de semana, tener claro los mecanismos de control de las actividades realizadas, y resultados esperados de acuerdo a los objetivos trazados.

Tal es el caso como lo enuncian Ramos, Ramos-Galarza y Tejera (2020): en los resultados de su investigación se pudo determinar que el teletrabajo es positivo en cuanto a la productividad siempre que el teletrabajador disponga de recursos y condiciones adecuadas, capacidades para el desarrollo de las actividades y la forma como se desarrolla el trabajo desde el domicilio. De modo pues, todos estos aspectos deben estar bien determinados en el protocolo o directrices del teletrabajo.

En razón a lo antes expuesto, Giniger (2020): describe que, la jornada de trabajo efectivamente se extendió con la pandemia, no tiene un inicio ni un final, desde el comienzo del día hasta finalizar el trabajador o empleado que se desempeña en teletrabajo está realizando sus actividades e incluso en horas nocturnas o de descanso suelen levantarse para realizar alguna tarea, influyendo además en las actividades del hogar sobre todo en el caso de las mujeres que llevan esta responsabilidad y cuyas consecuencias serán drásticas para su salud.

En tal sentido, el teletrabajo en el contexto de la pandemia requiere que la autoridad pública o el empleador del sector privado de la Institución educativa definan de forma precisa las directrices para el desarrollo de las actividades mediante teletrabajo, por cuanto, la forma abrupta e inesperada en la que muchas empresas e instituciones comenzaron a implementar esta figura jurídica, aunado a las restricciones generadas por la pandemia, no permiten la práctica adecuada del mismo, aun cuando, se han realizado los mayores esfuerzos y de cierto modo se han superado muchas barreras, obteniendo resultados favorables sobre todo en el sector educativo, a largo plazo podrán 
ser notorios los efectos negativos de la pandemia en la ejecución inadecuada de este modo de organización del trabajo donde se laboran jornadas prolongadas, con sobre carga de trabajo y sin establecer límites entre las la vida personal, familiar y social del teletrabajador.

\section{Conclusiones}

La pandemia originada por el COVID-19 hizo necesaria la implementación del teletrabajo a nivel global para continuar impartiendo la educación en la sociedad, lo cual como derecho humano fundamental y elemento esencial para el desarrollo de un país no podía detenerse ante la incertidumbre de una enfermedad altamente contagiosa y que aún permanece causando graves daños a la salud, la economía, el mercado laboral y a la población en general.

Debido a la forma inesperada en la que se presentó la emergencia sanitaria algunos estados de la región no estaban preparados para la aplicación de este modo de empleo, en aspectos laborales, sociales, tecnológicos y jurídicos, lo que hizo necesaria la promulgación de normas o reformas de las legislaciones ya existentes, así como, la toma de medidas por parte de autoridades del sector público y empleadores del sector privado para ajustarse al contexto del COVID-19 e implementar el teletrabajo de la manera más adecuada, siendo este el caso particular del sector educativo a nivel universitario.

En el ámbito educativo el reto fue aún mayor desde muchos aspectos, ya que se ven involucrados en la práctica del teletrabajo Institución, docentes, y estudiantes, donde la educación a nivel global se imparte de forma presencial y no a distancia, razón por la cual la efectividad y eficacia de este modo de trabajo debe medirse no sólo desde la perspectiva del docente teletrabajador y la Institución que controla y supervisa las actividades desempeñadas por el docente, sino también, considerar los resultados sobre los estudiantes que 
están recibiendo la educación en línea o a distancia a través de este sistema, a fin de conocer la aceptación de los mismos sobre esta nueva modalidad del proceso de enseñanza aprendizaje, así como, las barreras o dificultades que se han presentado, lo que lleva a la necesidad de realizar investigaciones bajo este enfoque, a fin de establecer propuestas, y medidas científicas que permitan ejecutar acciones efectivas para el beneficio de todos los actores sociales que se desempeñan en teletrabajo.

Aunado a lo anterior, el teletrabajo ejecutado en el sector educativo en general y particularmente a nivel universitario en tiempos de pandemia, requiere de un trato específico, es decir, no puede ser considerado como el teletrabajo planteado antes de la pandemia del COVID-19, donde no existían restricciones en el ámbito laboral, económico y social, por tanto, para prevenir consecuencias negativas a futuro en sus resultados, tanto para la Institución, como para el docente y estudiantes es menester el establecimiento de directrices claras y precisas para su desarrollo, que traten los aspectos laborales, tecnológicos, sociales, familiares y personales, ya que sus efectos frente a la pandemia repercuten no sólo en los actores involucrados, sino en la vida personal y en el grupo familiar del docente teletrabajador.

A tales efectos, es importante continuar con investigaciones sobre el teletrabajo frente a la emergencia sanitaria generada por el COVID-19 a fin de determinar si las repercusiones del coronavirus han afectado los derechos laborales de los trabajadores que se desempeñan en teletrabajo, así como identificar los factores que han limitado el desempeño de los docentes en teletrabajo.

\section{Referencias}

Academia Internacional de Teletrabajo (2017). Primer Informe: Estado del

Teletrabajo en América Latina y el Caribe. Capítulo América Latina y el Caribe. Brasil: ITA-LAC. 
Acuña, M., \& Sánchez, C. (2020). Educación Superior pospandemia. Las asimetrías de la brecha tecnológica. RGV: Revista Venezolana de Gerencia, 25(92), 1282-1287, e-ISSN: 1315-9984. Recuperado de: https://doi.org/10.37960/rvg.v25i92.34304

Castellano, J., Almagro, J., \& Fajardo, Á. (2021). Percepción estudiantil sobre la educación online en tiempos de COVID-19: Universidad de Almería (España). Revista Scientific, 6(19), 185-207, e-ISSN: 25422987. Recuperado de:

https://doi.org/10.29394/Scientific.issn.2542-2987.2021.6.19.9.185-207 CETyFH-UV (2020). Guía para el Teletrabajo en condiciones de Pandemia COVID-19. Chile: Centro de Estudio del Trabajo y Factores Humanos de la Universidad de Valparaíso.

Conejo-Navarro, F. (2020). Teletrabajo Asistido en los tiempos del Coronavirus (COVID-19). Tecnología Vital, 2(8), 40-49, e-ISSN: 22155740. Recuperado de:

https://revistas.ulatina.ac.cr/index.php/tecnologiavital/article/view/392

Cóndor-Herrera, O. (2020). Educar en tiempos de COVID-19. CienciAmérica, 9(2), 31-37, e-ISSN: 1390-9592. Recuperado de:

http://dx.doi.org/10.33210/ca.v9i2.281

Chávez, J., Martínez, J., \& Dávila, R. (2020a,b,c). Educación a Distancia y Teletrabajo. Daena: International Journal of Good Conscience, 15(1), 264-277, e-ISSN: 1870-557X. México: Instituto de Estudios Superiores Spenta Mexico.

Estévez, A. (2020). Mapeo de actores en situación de teletrabajo: consecuencias organizacionales por la pandemia de 2020 en la Administración Pública Argentina. RVG: Revista Venezolana de Gerencia, 25(92), 1266-1270, e-ISSN 1315-9984. Recuperado de: https://produccioncientificaluz.org/index.php/rvg/article/view/34302

Giniger, N. (2020). Teletrabajo. Modalidad de Trabajo en Pandemia. Revista 
Observatorio Latinoamericano y Caribeño, 4(1), 23-39, e-ISSN: 18532713. Ciudad de Buenos Aires, Argentina: Universidad de Buenos Aires. Guaña-Moya, E., Quinatoa-Arequipa, E., \& Pérez-Fabara, M. (2017). Tendencias del uso de las tecnologías y conducta del consumidor tecnológico. Ciencias Holguín, 23(2), 1-17, e-ISSN: 1027-2127. Recuperado de: https://www.redalyc.org/articulo.oa?id=181550959002 MDT (2020a,b,c). Acuerdo Ministerial nro. MDT-2020-076 de fecha 12 de marzo. Quito, Ecuador: Ministerio del Trabajo.

Montes-Rodríguez, A., Chen-Quesada, E., Hernández-Sánchez, A., \& Villalobos-Benavides, V. (2020). Ruta de la gestión educativa del CIDE ante el contexto COVID-19. Revista Electrónica Educare, 24(Suplemento Especial), 1-4, e-ISSN: 1409-4258. Recuperado de: https://doi.org/10.15359/ree.24-S.7

Moreno, L. (2020). Decreto ejecutivo no. 1017. declárese el estado de excepción por calamidad pública en todo el territorio nacional, por los casos de coronavirus confirmados y la declaratoria de pandemia de COVID-19. Quito, Ecuador: Presidente Constitucional de la República.

OIT (2020). El teletrabajo durante la pandemia de COVID-19 y después de ella. Primera Edición, ISBN: 978-92-2-033092-0. Ginebra: Oficina Internacional del Trabajo. Organización Internacional del Trabajo.

Peralta, A., Bilous, A., Flores, C., \& Bombón, C. (2020). El impacto del teletrabajo y la administración de empresas. Recimundo: Revista Científica Mundo de la Investigación y el Conocimiento, 4(1), 326-335, e-ISSN: 2588-073X. Recuperado de:

https://recimundo.com/index.php/es/article/view/761

Quintero, J. (2020). El Efecto del COVID-19 en la Economía y la Educación: Estrategias para la Educación Virtual de Colombia. Revista Scientific, 5(17), 280-291, e-ISSN: 2542-2987. Recuperado de: 
https://doi.org/10.29394/Scientific.issn.2542-2987.2020.5.17.15.280-

$\underline{291}$

Ramos, V., Ramos-Galarza, C., \& Tejera, E. (2020). Teletrabajo en tiempos de COVID-19. Revista Interamericana de Psicología, 54(3), 1-29, eISSN: 2329-4795. Recuperado de:

https://doi.org/10.30849/ripijp.v54i3.1450

Risso, M., Ruocco, G., Ramos, B., Valentín, G., Slinger, S., Costa, L., ...Garat, M. (2020). EI Derecho frente a la pandemia por COVID-19. Revista de Derecho, (21), 1-24, e-ISSN: 2393-6193. Recuperado de: https://doi.org/10.22235/rd.vi21.2193

Sánchez, G., Montenegro, A., \& Medina, P. (2019). Teletrabajo una propuesta de innovación en productividad empresarial. Revista 593 Digital Publisher CEIT, 4(5-1), 91-107, e-ISSN: 588-0705. Recuperado de: https://doi.org/10.33386/593dp.2019.5-1.133

Tapasco-Alzate, O., \& Giraldo-García, J. (2020). Asociación entre posturas administrativas de directivos y su disposición hacia la adopción del teletrabajo. Información tecnológica, 31(1), 149-160, e-ISSN 07180764. Recuperado de:

http://dx.doi.org/10.4067/S0718-07642020000100149

Velásquez, C., \& Vera, M. (2018). Teletrabajo: Una Revisión Teórica sobre sus Ventajas y Desventajas. Investigatio, (10), 41-53, e-ISSN: 26028336. Recuperado de:

https://revistas.uees.edu.ec/index.php/IRR/article/view/194 


\section{Joanna Carolina Ramírez Velásquez \\ e-mail: joanna.ramirez@ucacue.edu.ec}

Nacida en el estado Zulia, Venezuela, el 20 de

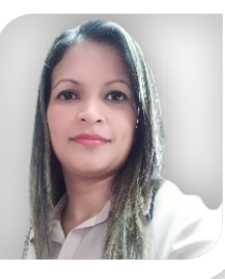
diciembre del año 1979. Abogada por la Universidad del Zulia (LUZ); Magister en Derecho Laboral y Administración del Trabajo, Mención Derecho Laboral por la Universidad del Zulia, Venezuela; Máster en Salud de los Trabajadores por la Universidad de Ciencias Médicas de la Habana (UCMH); Experiencia laboral previa como Abogada en el Instituto Nacional de Prevención Salud y Seguridad Laborales del Ministerio del Trabajo, Venezuela; actualmente me desempeño como docente en la Universidad Católica de Cuenca (UCACUE), extensión La Troncal, siendo responsable del Área de Investigación Formativa de la Carrera de Derecho. 


\section{Luis Ernesto Quinde Quizhpi \\ e-mail: lequindeq@ucacue.edu.ec}

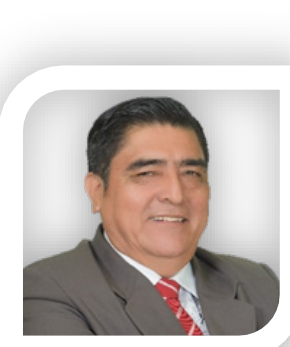

Nacido en Cuenca, Ecuador, el 5 de septiembre del año 1959. Abogado de los Tribunales de Justicia del Ecuador; especialista en Derecho Constitucional, por la Universidad de Especialidades Espíritu Santo (UEES); Magister en Derecho Constitucional, por la Universidad de Especialidades Espíritu Santo; doctorando en la Universidad Andina Simón Bolívar (UASB), de la República de Bolivia; actualmente docente de la Universidad Católica de Cuenca (UCACUE), extensión La Troncal, me desempeñé como Juez Temporal y como Notario Suplente. 


\section{Ricardo Agustín Alarcón Vélez}

e-mail: ricardo.alarcon@ucacue.edu.ec

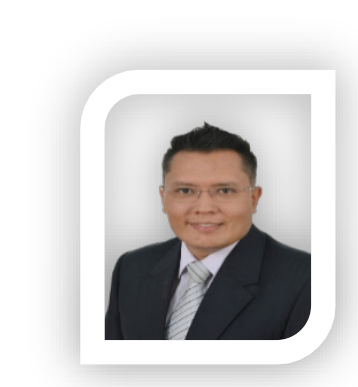

Nacido en Cuenca, Ecuador, el 1 de septiembre del año 1981. Doctor en Jurisprudencia y Abogado de los Tribunales de Justicia del Ecuador; Magister en Derecho Administrativo y en Derecho Constitucional, por la Universidad Técnica Particular de Loja (UTPL); actualmente docente de la Universidad Católica de Cuenca (UCACUE), extensión La Troncal, me desempeñe como Procurador Síndico Municipal, Registrador de la Propiedad. 


\section{Cesar Remigio Vega Abad \\ e-mail: crvegaa@ucacue.edu.ec}

Nacido en Cañar, Ecuador, el 7 el abril del año 1983.

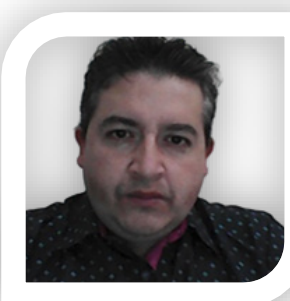

Estudie Ingeniería de Sistemas en la Universidad Católica de Cuenca (UCACUE); culminé mis estudios profesionales en la Maestría de Redes de Comunicaciones en la Pontificia Universidad Católica del Ecuador (PUCE); actualmente me desempeño como Docente en la Carrera de Derecho de la Universidad Católica de Cuenca (UCACUE), extensión La Troncal.

El contenido de este manuscrito se difunde bajo una Licencia de Creative Commons ReconocimientoNoComercial-Compartirlgual 4.0 Internacional 